\title{
Research on Standardization of Aircraft Battle Damage Description
}

\author{
Yang $\mathrm{Jia}^{1}$, Zhao Hanwu ${ }^{* *}$, and Guo $\mathrm{Kai}^{2}$ \\ ${ }^{1}$ Aviation Maintenance NCO Academy, Air Force Engineering University, 464000, Xinyang, China \\ ${ }^{2}$ Shijiazhuang Haishan Company, 050278, Shijiazhuang, China
}

\begin{abstract}
Rapid and accurate description of battle damage is the first step in aircraft battle damage repair (ABDR). The standardized description of damage information is the basis of the construction of aircraft battle damage data model. This paper studies and analyses the statistical description of battle damage in the view of definition, damage mode and cause classification of battle damage, so as to obtain the basis for standardized description of battle damage information.
\end{abstract}

\section{Introduction}

It is an important way to improve the sustained combat capability of aviation forces by repairing the damaged aircraft. Rapid and accurate assessment of battle damages is an important part of aircraft battle damage repair [1]. Due to the uncertainty of battle damage, the description of aircraft battle damage information presents the possibility of diversification compared with general fault information processing. In addition, while analyzing and processing aircraft damages, it is limited to the personnel level of information processing and analysis preference. In the digital era, the battle damage information without standardized processing will increase the degree of information fuzziness, so as to restrict the realization of related functions, and increase the difficulty of equipment reliability support for the future intelligent system applications such as data analysis system, expert evaluation system, and battle damage repair resource platform [2-4].

In order to solve such problems, provide support for data analysis system, expert evaluation system, battle damage repair resource platform and other system modeling, improve the confidence of data information, and carry out the research on digital auxiliary technology of aircraft battle damage repair, it is necessary to redescribe the information from the definition and elements of battle damage information without changing the nature of information. At the initial stage of information generation, the influence of human factors on information quality is eliminated, and the data information is transformed into data resources that can guide the later work and can be used for quantitative analysis.

\section{Definition of aircraft battle damage}

In GJB 3897-1999, technical manual preparation requirements for aircraft battle damage assessment and repair [5], the definition of aircraft battle damage is described as "battle damage, random failure, consumable failure, human error, accident, shortage of maintenance supplies and environmental change and other events that hinder the aircraft from completing the scheduled tasks in wartime".

Among them, "random fault, consumable fault, human error" have been studied in daily maintenance $[6$, 7], and the related statistical description has standardized requirements and corresponding research, which are not discussed in this paper. This paper mainly studies the mode and cause classification of battle damages and accidents. Before mode and cause discuss, we firstly focus on the elements classification of aircraft battle damage information.

\section{Elements of aircraft battle damage information}

According to the definition, combined with the actual situation and processing process, the aircraft battle damage information can be divided into basic damage information, battle damage presentation, cause analysis, treatment measures, processing results and so on [6].

\subsection{Basic information}

Basic information of battle damage includes damage date, place, affiliated unit, aircraft type, number, crew, etc. these type of information usually are in fixed formats and well known [7]. This type of information always has strict format requirements.

\subsection{Damage appearance}

Damage appearance means the presentation of battle damage. It includes the damaged system/subsystem,

\footnotetext{
* Corresponding author: yeaper@126.com
} 
name of damaged parts, model of damaged parts, mode of battle damages, and specific phenomenon of damaged aircraft or system [8].

\subsection{Causes}

The causes of damage need to be analyzed. It includes the classification of the causes of battle damages, the specific causes of battle damages, and the analysis process [9].

\subsection{Treatment methods}

The treatment methods include repair methods, necessary supporting equipment / tools and inspection methods.

\subsection{Results}

The treatment results includes man-hour, processing total time, environment conditions, aircraft restrictions, etc.

\section{Standardized classification of battle damage information}

The normalization of damage information description is to standardize the five elements of damage information. Through the analysis, the description can be divided into three categories for normalization $[6,10]$.

\subsection{Type $A$ information}

Type A information means that there is no need to analyze and define the format or content separately. It mainly includes damage date, unit, aircraft and related systems. This type of information has strict format requirements. It is not allowed to fill in the information with correct content but wrong format [11].

\subsection{Type B information}

Type B information means that there is no need to fill in the content of standardization. It mainly includes the consequence of battle damage, the specific phenomenon of damage and the cause of damage. This type of information is mainly explanatory information, which requires detailed and accurate description, clear organization, clear logic and clear follow-up.

\subsection{Type $C$ information}

Type $\mathrm{C}$ information means that detailed and standardized definition is needed. It mainly includes the mode of battle damage and the classification of its causes [12]. The division of these two aspects will be described in detail below.

\section{Standardization of battle damage mode}

Considering the urgency of carrying out battle damage repair in battlefield environment, the description of aircraft battle damage mode should meet the following requirements [14-16]:

1) Brevity: it is not necessary to note the damage mode when it is not necessary.

2) High efficiency: in all damage categories, no more than four comparative analyses are required to identify a particular damage.

3) Easy to identify: when confirming the mode of a certain damage, multiple modes cannot be selected in $70 \%$ of cases.

4) Low interference: in $90 \%$ of cases, different people cannot have different understanding of the damage mode.

In practical use, the convenience of analysis should be fully considered, and the division of battle damage modes must be reasonable. If the division is too detailed, it will be difficult to use, and if too few, the boundary will be unclear. There are two main approaches to the study of aircraft damages.

According to literature $[1,17]$, the battle damage modes were divided into the following 19 categories: grooving, spalling, performance change, pit, hole, deformation, crack, surface damage, burn, fracture, notch, wear, loose connection, sticking, open circuit, short circuit, poor contact, biological damage and soft damage.

In reference $[8,18]$, the battle damage modes were divided according to different causes (such as fragments, projectiles, burns, etc.), different materials (composite damage, organic glass damage, etc.), structure / location and damage degree (large-area deformation, small-area damage of a certain part, etc.) [19].

The two classification methods above both have limitations in practice. For example, the classification method in literature [1] has the following limitations: 


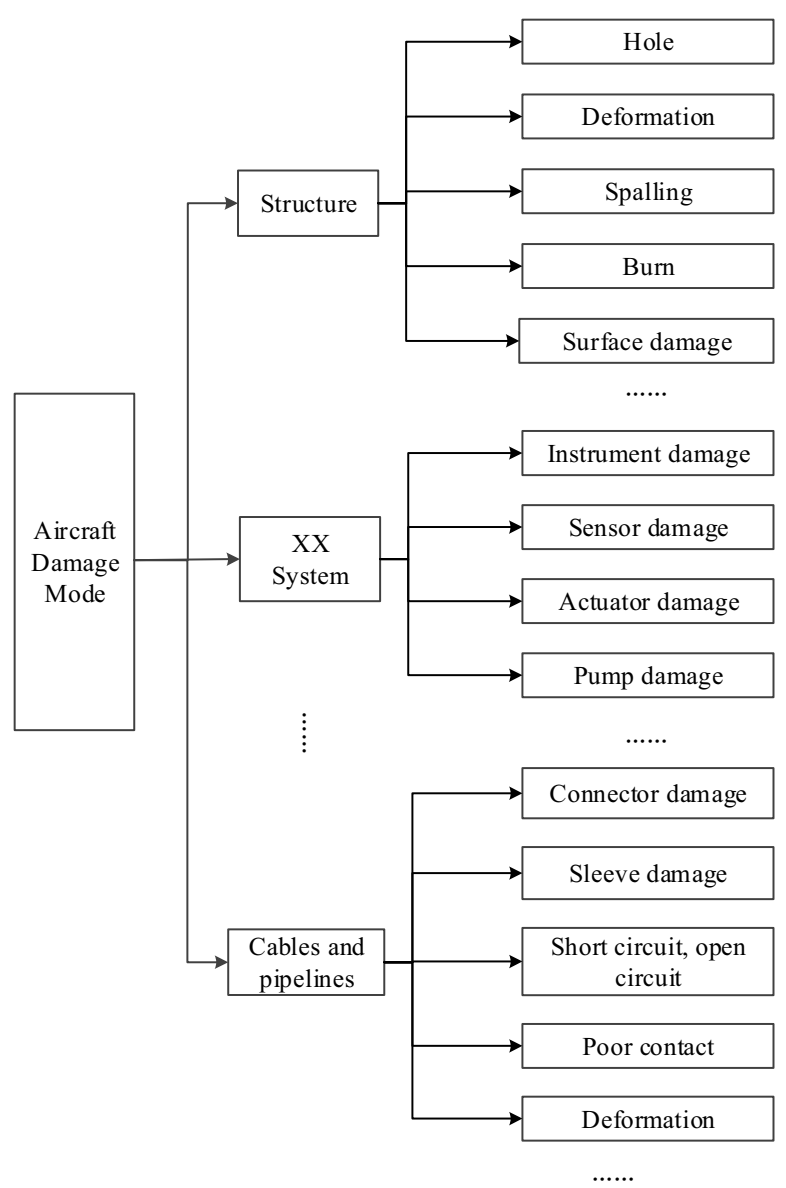

Fig. 1. Classification of aircraft battle damage modes

First of all, classification is not easy to identify, such as pit and deformation, groove and crack, etc., which is easy to cause ambiguity and cause unnecessary interference.

Secondly, the names of some battle damage modes are not clear enough, and different people may have different understandings.

Moreover, the classification does not cover all battle damages. For the damage of aircraft functional system, cable line and hydraulic pipeline, the existing classification fails to cover.

At the same time, the classification methods in reference [8, 20-22] are not clear and easy to identify.

Based on the further analysis of the battle damage and repair needs of aircraft, the battle damage modes can be divided into three categories: airframe structure, airborne system and cable / pipeline according to different major specialties, and then subdivided according to the specific damage forms. The proposed classification of battle damage patterns is shown in Fig. 1 above.

According to the classification above, we classify the cable damage into six kinds below in Fig. 2. The 6 kinds of damage mode are "cable cut off", "insulation burned", "sleeve worn", "shield sleeve worn", "connector broken" and "connector clamp bent" [11].

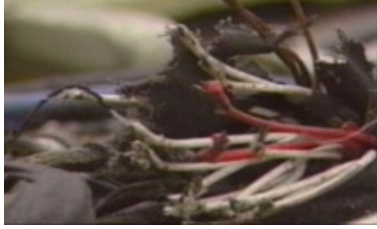

Cable cut off

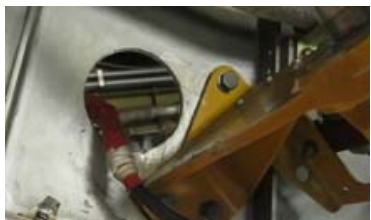

Cable sleeve worn

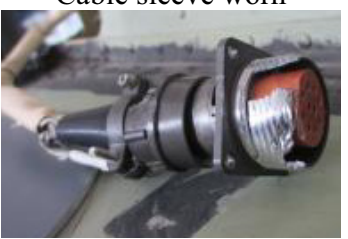

Cable connector broken

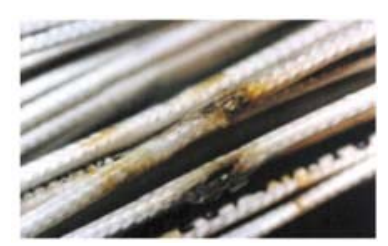

Cable insulation burned

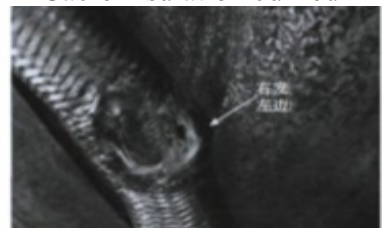

Cable shield sleeve worn

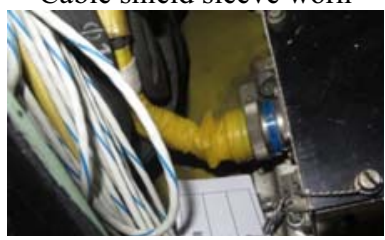

Cable connector clamp bent

Fig. 2. Examples of cable damage classification

\section{Standardization of causes of battle damages}

The causes of battle damages can be divided into the following categories:

Direct damage. It is caused by shrapnel and shock wave; the appearance characteristics of this kind of damage are obvious and easy to judge, and the main manifestation is through damage, deformation and spalling.

Indirect damage. It means chemical, impact, secondary, airframe fatigue, etc.; this kind of damage is often caused by direct damage transmission, or because of the bad combat environment, or because of the increase of combat intensity, resulting in airframe fatigue, pilot's failure operation, etc.

Burn damage. It's the damage of the aircraft structure and internal systems caused by fire and overheating in the air or on the ground; the manifestations of such damage are various, which may not only cause the deformation of aircraft structure or surface coating damage, but also cause the failure of internal cable pipelines and system components.

Other damage. It includes the damages caused by new concept weapons, such as graphite bomb, electromagnetic pulse weapon, nuclear weapon, etc.

\section{Results and discussion}

With the help of the standardization of the above classification methods, a standardized description of the aircraft battle damage case in practice is analyzed. The following are two actual cases [23].

\subsection{Case one}

Here are two descriptions of battle damages, one of which is "One actuator and two hydraulic hoses in the hydraulic chamber were damaged" and the other is "The 
oil inlet pipe joint of the flat tail's integrated control valve was slightly leaking." According to the descriptions, the two damages can be considered as two completely different types. One belongs to "damaged hose" and the other belongs to "oil leakage". Without standardization, the damage mode of the first may be described as "hose damage", "hydraulic hose's hole", "actuator hose crack", "hydraulic hose failure", etc.; and the other maybe described as "oil leakage", "pipeline leaking", "control valve damage" and so on. However, after researching and analyzing on the damage mode and description standardization, the damage mode of both cases will be determined as "allowable leakage". They both belong to the same damage mode [24-25].

Hence, two seemingly irrelevant cases of battle damage are determined to be of the same type after the analysis of the damage mode.

\subsection{Case 2}

A certain aircraft battle damage information once was described as "disconnected to the automatic flight control system was found during air combat." Through the analysis of the damage, it is concluded that the source of the problem is not the failure of the automatic flight control system, but the damage of the angle of attack sensor of the avionics system [26-28]. Therefore, the damage mode should actually be "equipment damage abnormal electronic equipment - abnormal operation of flight control equipment - abnormal response - unstable operation - data jumping" instead of "abnormal response abnormal disconnection" [29].

\section{Conclusions}

One of the most important characteristics of aircraft battle damage repair, which is different from peacetime maintenance, is its time cost limitation on battle damage assessment and repair. Standardizing the description information of aircraft battle damage not only helps to reduce the time cost in battle damage assessment, but also helps to promote the application of remote assessment, expert assessment and intelligent assessment. In view of the difference of external threat sources, combat environment and the diversity of aircraft battle damage, the research on the standardization of battle damage mode and causes classification has not yet finished, and there will be a long distance before it reaches practice level.

\section{References}

1. J. Zhang, et al. Battle damage repair of aircraft structure (National Defense Industry Press, 2007)

2. R. Xue, S. Li. (2003) Research on decision support system for aircraft battle damage assessment and repair. Modern electronic technology, 20: 40-42

3. P. Zhao, B. Ji, H. Wei, J. Dai, F. Hu. (2014) A brief introduction of modeling and simulation in the research of aircraft battle damage repair. Electronics
Optics \& Control, 21, 02: 55-59

4. W. Yao, K. Cai, P. Zhou, L. Hu. (2011) Research on information management and decision making system for aircraft battle damage repair. New Technology \& New Process. 09: 60-62

5. GJB 3897-1999, Requirements on compilation of technical manual for aircraft battle damage assessment and repair [S]

6. Z. Zhou. (2018) Research on normalization of fault information description. Aviation Maintenance \& Engineering, 07: 70-72

7. X. Mu, Y. Zhong. (2016) Discussion on standardization of maintenance records. Science and Technology \& Innovation, 01: 155

8. J. Zhang, et al. Aircraft battle damage repair engineering (Aviation Industry Press, 2007)

9. W. Yao, K. Cai, P. Zhou, K. Yu. (2012) Research on basic forms of aircraft battle damage. New Technology \& New Process, 04: 47-49

10. K. Cai. (2015) Evaluation and hazard analysis of aero-engine battle damage. In: Proceedings of the 2nd China Aviation Science \& Technology Conference. Beijing, pp. 143-147

11. H. Zhao, T. Zhang, M. Hao and J. Yang. (2020) Research on Military Aircraft Electrical Wiring System Damage Mode and Battle Damage Assessment and Repair. Journal of Air Force Engineering University (Natural Science Edition), 21(5): 106-111

12. H. Zhao, F. Sun, J. Yang. (2019) Research on MILDTL-87158 and the enlightenment to military aircraft battle damage repair. 297(06):23-26

13. NATO RTO Technical Activity Proposal (TAP) AVT-144, October 2004

14. NATO ARMP-7, "NATO R\&M Terminology", Edition 1, July 2001

15. AAP-6 NATO Glossary of Terms and Definitions, 2008

16. L.A. Kratz, R.T. Fowler, J.D. Cothran, (2002) Achieving Defence Transformation Through Total Life - Cycle System Management, Project Management (PM) Magazine, Vol. XXXI, No. 5:2327

17. United States Government Accountability Office GAO Report GAO-03-300. (2003) Military Readiness - DoD Needs a Clear and Defined Process for Setting Aircraft Availability Goals in the New Security Environment

18. K. Hart, (2006) Understanding Our Aging Aircraft Fleets: Deriving Aging Aircraft Requirements from AF Maintenance Metrics and Data, presentation at 9th Joint FAA/DoD/NASA Conference on Aging Aircraft

19. US Air Force Instruction AFI 21-101, Aircraft and Equipment Maintenance Management, Section 1.15.3 Primary Maintenance Metrics, 29 June 2006

20. US Air Force Instruction AFI 21-103, Equipment 
Inventory, Status and Utilization Reporting, Attachment 2 Maintenance Status Codes and Condition Status Codes, 14 December 2005

21. H.S. Balaban, R.T. Brigantic, S.A. Wright, A. F. Papatyi, (2000) A Simulation Approach to Estimating Aircraft Mission Capable Rates for the United States Air Force, In: Proceedings of the 2000 Winter Simulation Conference

22. FM 4-30.31 (FM 9-43-2), Recovery and Battle Damage Assessment and Repair. 2006

23. Air Force Research Laboratory, (1997) Air Vehicles Directorate Software Aids in A-10 Maintenance Issue. Wright-Patterson Air Force Base Newsletter

24. K. CAI, et al. (2010) Spare Part Requirement Prediction Method for Aircraft Battle Damage Repair. Electronics Optics \& Control, 12: 123-128

25. Q. Pan, L. Xiong, X. Liu. (2007) Design and development of composite aircraft battle damage repair simulation system. Acta Armamentarii, 11: 25.

26. B. Kaplan, J. Wallick. (1994) Aircraft Battle Damage Repair (BDR) Analysis Methodology Development Requirements. Logistics Management Inst McLean VA, 21:201-204

27. R. Bartholomeusz, P. Pearce, R. Vodicka. (2002) Rapid application technology: Aircraft battle damage repairs. In Advances in the bonded composite repair of metallic aircraft structure 21 : 761-781

28. Crum, K., Dierker, R., Grody, J., Thomas, D. L., \& Clark, M. E. (2000). Aircraft Battle Damage Assessment and Repair (ABDAR)

29. M. Hou, S. Li, et al. (2006). Evaluation of aircraft battle damage repair capability based on gray correlation-hierarchical analysis. Electronics Optics \& Control. 4:35-39 